\title{
Enhancing Förster nonradiative energy transfer via plasmon interaction
}

\author{
L. J. Higgins $s^{\text {a }}$ X. Zhang ${ }^{\text {a }}$, C. A. Marocico ${ }^{\text {a }}$, G. P. Murphy ${ }^{\mathrm{a}}$, V. K. Karanikolas ${ }^{\mathrm{a}}$, Y. K. Gun'ko ${ }^{\mathrm{b}}$, V. \\ Lesnyak $^{\mathrm{c}}$, N. Gaponik ${ }^{\mathrm{c}}$, A. S. Susha ${ }^{\mathrm{d}}$, A. L. Rogach ${ }^{\mathrm{d}}$, P.J. Parbrook ${ }^{\mathrm{e}}$ and A. L. Bradley*a

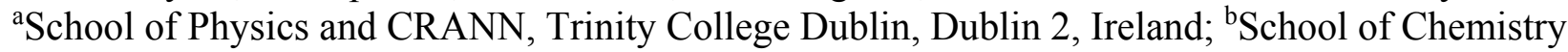 \\ and CRANN, Trinity College Dublin, Dublin 2, Ireland; 'Physical Chemistry, TU Dresden, \\ Bergstrasse 66b, 01062 Dresden, Germany; ${ }^{\mathrm{d} D e p a r t m e n t}$ of Physics and Materials Science \& Centre \\ for Functional Photonics (CFP), City University of Hong Kong, Hong Kong, China; ${ }^{\mathrm{e} T y n d a l l}$ \\ National Institute and School of Engineering, University College Cork, Lee Maltings, Prospect Row, \\ Cork, Ireland
}

\begin{abstract}
Plasmon-enhanced nonradiative energy transfer is demonstrated in two inorganic semiconductor systems. The first is comprised of colloidal nanocrystal CdTe donor and acceptor quantum dots, while the second is a hybrid InGaN quantum well-CdSe/ZnS quantum dot donor-acceptor system. Both structures are in a planar geometry. In the first case a monolayer of Au nanospheres is sandwiched between donor and acceptor quantum dot monolayers. The largest energy transfer efficiency is seen when the donor is $\sim 3 \mathrm{~nm}$ from the Au nanopshere. A plasmon-enhanced energy transfer efficiency of $\sim 40 \%$ has been achieved for a separation of $3 \mathrm{~nm}$ between the Au nanopshere monolayer and the acceptor monolayer. Despite the increased energy transfer efficiency these conditions result in strong quenching of the acceptor QD emission. By tuning the Au nanosphere concentration and Au nanosphere-acceptor QD separation the acceptor QD emission can be increased by a factor of $\sim 2.8$. The plasmon-enhanced nonradiative energy transfer is observed to extend over larger distances than conventional Forster resonance energy transfer. Under the experimental conditions reported herein, it can be described by the same $d^{-4}$ dependence but with a larger characteristic distance. Using a Ag nanobox array plasmonic component plasmon-enhanced nonradiative energy transfer has also demonstrated from an InGaN quantum well to a $\sim 80 \mathrm{~nm}$ thick layer of $\mathrm{CdSe} / \mathrm{ZnS}$ colloidal quantum dots. An efficiency of $\sim 27 \%$ is achieved, with an overall increase in the QD emission by $\sim 70 \%$.
\end{abstract}

Keywords: Plasmonics, nonradiative energy transfer, Förster resonance energy transfer, FRET, plasmon-enhanced nonradiative energy transfer, localised surface plasmons, quantum dots and quantum wells

\section{INTRODUCTION}

Nonradiative energy transfer by fluorescence resonance energy transfer, also known as Förster resonance energy transfer, is extensively used to measure interactions in biology and chemistry. It is routinely employed in bioimaging and sensing systems using fluorescent molecules. Arising from the nearfield interaction of a donor dipole and acceptor dipole, this nonradiative energy transfer mechanism has a well-known $\mathrm{r}^{-6}$ distance, which can be exploited for a spectroscopic ruler. ${ }^{1,2}$ Advances in the synthesis and fabrication of nanoscale emitters has motivated extensive study of nonradiative energy transfer in a variety of other materials systems. The donor and/or acceptor can be, for example, a quantum dot (QD), quantum wire, quantum well (QW), 2D material (such as graphene or $\mathrm{MoS}_{2}$ ) or metallic nanoparticle. Such systems are relevant to a myriad of potential applications in enhanced sensing, light harvesting, down-conversion light emitting diodes and optical computing. It has been shown that fluorophore-metal nanoparticle pairs exhibit large quenching distances, which can yield higher sensitivity detection for sensing applications. ${ }^{3}$ In the case of light harvesting and light emitting applications the nonradiative energy transfer mechanism can be used to transport energy from one part of the electrical device to another, overcoming issues with poor carrier conduction in some of the component materials. ${ }^{4}$

*bradlel@tcd.ie; phone+353-1-8963595, tcd.ie 
In more recent times, researchers have explored the possibility to control key signatures of nonradiative energy transfer between two fluorophores, namely the rate, efficiency and spatial extent, by engineering the local environment of the donor-acceptor pair. This can be achieved, for example, by placing the donor-acceptor pair in a cavity or in proximity to metallic structures..$^{5-8}$ The influence of metallic plasmonic structures on the emission properties of proximal dipoles continues to be intensively investigated, with large increases in the spontaneous emission rate and enhanced directionality of the emission reported. ${ }^{9}$ Plasmon-mediated nonradiative energy transfer between two fluorophores has been reported in a variety of geometries including core-shell structures, planar structures and single donor-acceptor pairs. ${ }^{10-13}$ Demonstrations of enhancement of the energy transfer rate, efficiency and distance have been achieved. However, the form of distance dependence and relative position of the metallic nanostructures, remained to be explored. Furthermore, for some applications, such as light emitting diodes, the emission of the acceptor is a critical parameter. Therefore, it is important to consider not only the conditions for optimizing the acceptor pumping via nonradiative energy transfer but also the impact on the acceptor emission.

In this paper, we look at studies on two semiconductor nonradiative energy transfer systems in planar geometries. The first is comprised of monolayers of nanocrystal quantum dot donors and acceptors, and where the plasmonic component is a monolayer of $\mathrm{Au}$ nanospheres. This flexible systems allows for arbitrary positioning of the three constituent components, providing insight into the distance dependence of the plasmon-enhanced nonradiative energy transfer process and the optimal position of the plasmonic component. ${ }^{14,15}$ The concentrations of nanoparticles in the three layers can also be tuned. Of particular interest are the dependences of the nonradiative energy transfer efficiency and the acceptor emission on the Au nanosphere concentration. Importantly, it is observed that conditions for the greatest energy transfer efficiency do not correspond to large enhancement of the acceptor emission. As mentioned above, exploitation of plasmon-enhanced nonradiative energy transfer for applications, such as light harvesting and light emitting diodes, requires being able to implement the mechanism in other material systems. Energy down-conversion from quantum wells to QDs is of particular interest for light-emitting diode applications, and this is the second planar semiconductor system reported on in this paper. In this system the plasmonic component is an array of lithographically fabricated Ag nanoboxes. Plasmon-enhanced nonradiative energy transfer from the QW to a layer of QDs has again been demonstrated, with an accompanying increase in the acceptor emission.

\section{RESULTS AND DISCUSSION}

\subsection{Plasmon-enhanced non-radiative energy transfer in quantum dot structures}

Many theoretical studies have reported on the impact of surface plasmons on nonradiative energy transfer, in particular the distance, angle and spectral dependences. ${ }^{8,16}$ These studies inform the design of the experimental system. The influence of an Au nanosphere on the nonradiateve energy transfer efficiency has been numerically investigated using a Green's tensor formalism. ${ }^{16}$ The donor quantum emitter emission and acceptor quantum emitter absorption are overlapped, and can vary from $400 \mathrm{~nm}$ to $700 \mathrm{~nm}$. Fig. 1(a), shows the dependence of the energy transfer efficiency on the size of the $\mathrm{Au}$ nanosphere, where two quantum emitters are positioned at $3 \mathrm{~nm}$ on opposite sides of the Au nanosphere. By varying the Au nanosphere size the spectral position of the localized surface plasmon resonance is tuned. The largest enhancements of the plasmon mediated energy transfer are observed for Au nanospheres with radii less than $10 \mathrm{~nm}$ and emission in the region of $550 \mathrm{~nm}$.

An experimental system comprised of CdTe nanocrystal quantum dots and a $5.5 \mathrm{~nm}$ diameter Au nanosphere is constructed using the layer-by-layer (LbL) assembly technique. A planar multilayer structure, comprised of monolayers of donor and acceptor CdTe colloidal nanocrystal QDs separated from each other by an intermediate monolayer of Au nanospheres, is constructed on a quartz substrate. The donor QDs with a $2.6 \mathrm{~nm}$ diameter have a lower peak emission wavelength of $547 \mathrm{~nm}$, than the larger acceptor QDs with a diameter of $3.4 \mathrm{~nm}$ and peak emission wavelength of 660 $\mathrm{nm}$. The CdTe QDs are stabilized by thiolglycolic acid which imports a negative surface charge. The $5.5 \mathrm{~nm}$ diameter colloidal $\mathrm{Au}$ nanospheres were stabilized by 4-dimethylaminopyridine (DMAP) and had a positive surface charge. Polyelectrolyte (PE) spacer layers, formed using bilayers of oppositely charged polyelectrolytes, namely, positively charged poly(diallyldimethylammonium chloride) (PDDA) and negatively charged poly(sodium 4-styrene sulfonate) (PSS), are used to control the separation between these three components of the plasmon-mediated energy transfer system. For reference donor-acceptor QD bilayers, QD-Au NP bilayers and QD monolayers bilayer structures are also prepared. 
The QD size and concentrations in the monolayers are determined from the absorption spectra, measured with a double beam UV-Vis Spectrometer (Shimadzu UV-2401 PC). ${ }^{17,18}$ The QD emission is characterized by measuring the PL spectra and time-resolved PL decays. The PL spectra were recorded with $400 \mathrm{~nm}$ excitation provided by a Xenon lamp. The time-resolved PL decays were recorded over an area of $80 \times 80 \mu^{2}(150 \times 150$ pixels $)$ using a PicoQuant Microtime200 time-resolved confocal microscope system with 150 ps resolution. Picosecond excitation pulses, at a wavelength of $470 \mathrm{~nm}$, were provided by a LDH-480 laser head controlled by a PDL-800B driver (PicoQuant). The donor and acceptor QD monolayer PL and absorption spectra, along with the Au nanosphere extinction spectrum is shown in Fig. 1(b). The Au nanosphere extinction spectrum shows a localised surface plasmon resonance at $532 \mathrm{~nm}$, with good overlap with the donor emission spectrum.
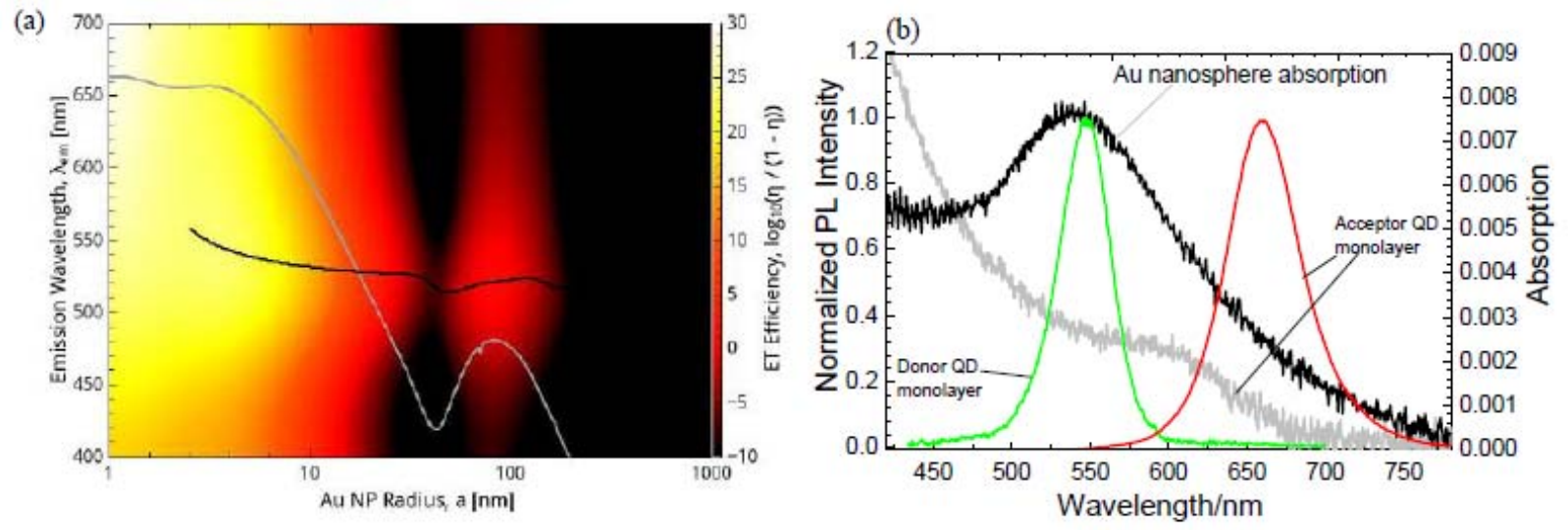

Figure 1. (a) A contour plot of the energy transfer efficiency as a function of the nanosphere size and central wavelength of the donor emission and acceptor absorption, where the donor and acceptor are both placed at $3 \mathrm{~nm}$ from the Au nanosphere. The light grey line shows the energy transfer (ET) efficiency for a wavelength of $525 \mathrm{~nm}$, while the black line represents the wavelength of the maximum ET efficiency as a function of the Au nanosphere size. Note in this plot the ET efficiency is represented as $\log _{10}(\eta /(1-\eta))$, where $\eta=k_{E T} /\left(k_{r}+k_{n r}+k_{E T}\right)$ with $k_{r}, k_{n r}$, and $k_{E T}$ corresponding to the radiative recombination rate, nonradiative recombination rate and energy transfer rate, respectively, for the donor-Au nanosphere-acceptor system. (b) Normalised PL spectra of the donor (green) and acceptor QD (red) QD monolayers. The acceptor QD monolayer absorption spectrum (light grey) and extinction spectrum of the Au nanosphere monolayer (black) are also shown.

The TRPL decays of trilayer and bilayer structures provide evidence of the signature of plasmon-enhanced non-radiative energy transfer, shown in Fig. 2. The decays are fitted with an amplitude weighted bi-exponential, and the average lifetime is quoted. Schematics of the structures are also shown in Fig. 2(b). In donor-acceptor bilayer the separation of 21 $\mathrm{nm}$ between the donor and acceptor monolayers is too large for any nonradiative energy transfer to be observed. In the trilayer structure the Au nanosphere layer is separated by $3 \mathrm{~nm}$ and $12 \mathrm{~nm}$ from the donor and acceptor quantum dot monolayers, respectively. In the case of the donor-Au nanosphere bilayer structure the donor lifetime is reduced to $\tau_{D o n-A u}$ $=(2.8 \pm 0.2) \mathrm{ns}$ compared with the donor-acceptor bilayer reference of $\tau_{D o n-A c c}=(3.7 \pm 0.2) \mathrm{ns}$. This is due to direct energy transfer from the donor QDs to the Au nanospheres. In the trilayer the donor lifetime is further reduced to $\tau_{D o n-T r i}$ $=(2.24 \pm 0.06) \mathrm{ns}$, indicating the presence of the additional decay path due to plasmon-enhanced nonradiative energy transfer to the acceptor QD monolayer. An experimental plasmon-enhanced energy transfer efficiency, $\eta$, of $\sim 20 \%$ is calculated using

$$
\eta=1-\frac{\tau_{D o n-T r i}}{\tau_{D o n-A u}}
$$

Fig. 2 (b) shows the PL spectra for the reference bilayer, the donor-Au nanosphere bilayer and the trilayer. Comparing firstly the reference bilayer with the trilayer structure with a $3 \mathrm{~nm}$ donor-Au nanosphere separation, an enhancement of the acceptor emission is observed, even though both structures have a similar donor-acceptor separation of $\sim 21 \mathrm{~nm}$. It is also noted that this enhancement cannot be attributed directly to the interaction between the acceptor QDs and the Au nanospheres as the acceptor-Au nanosphere bilayer shows quenched acceptor emission relative to the reference bilayer. 
It is also evident that the donor emission is further quenched compared to the donor-Au nanosphere bilayer, as expected from the PL decays discussed above. Therefore, the increased emission in the trilayer structure is attributed to plasmonenhanced energy transfer. In addition it can be noted that this enhancement is very sensitive to the donor-Au nanosphere separation. As this separation is increased the acceptor emission quickly collapses to the level observed for the acceptor$\mathrm{Au}$ nanosphere bilayer, indicating that small donor-Au nanosphere separations are optimal for enhancing the acceptor emission.
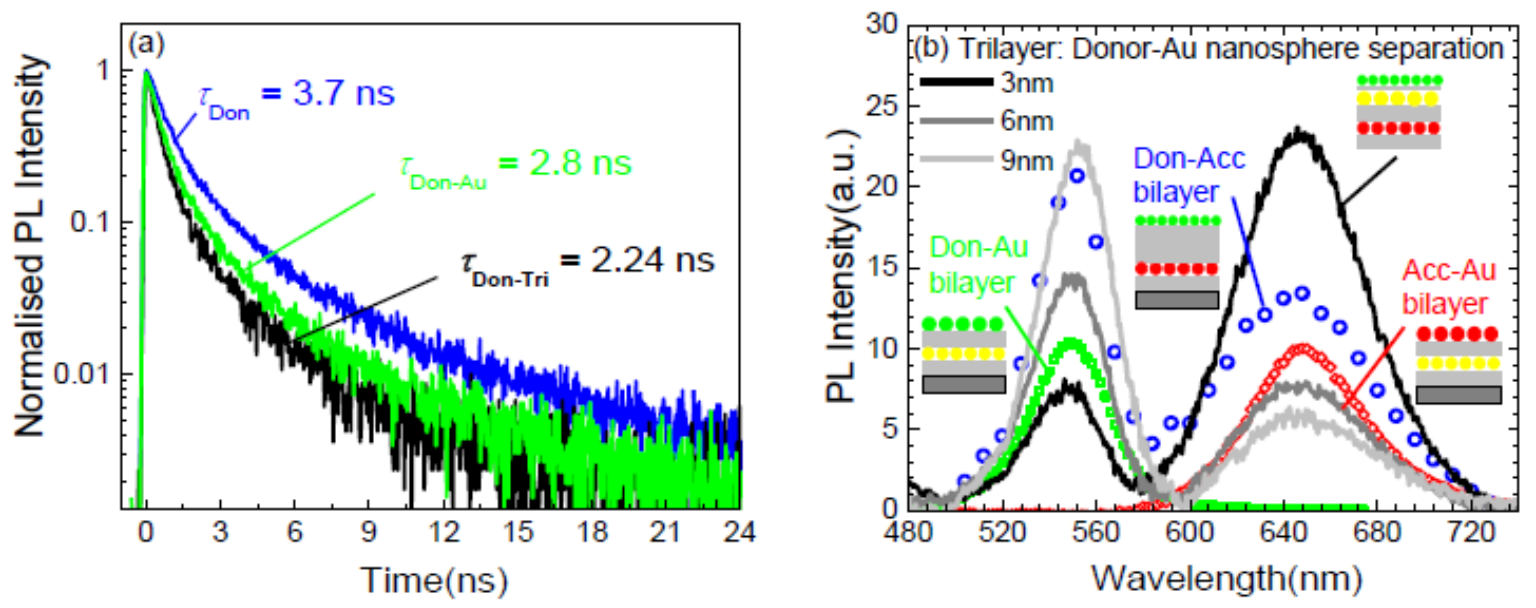

Figure 2. (a) Normalised time-resolved donor PL decays for the reference donor-acceptor bilayer (blue), donor-Au nanosphere bilayer (green) and trilayer structure (black). (b) PL spectra of the donor-acceptor bilayer reference with a donor-acceptor separation of $21 \mathrm{~nm}$ (blue circles), and the acceptor-Au nanosphere (red diamonds) and donor-Au nanosphere (green squares) bilayers with a separations of $12 \mathrm{~nm}$ and $3 \mathrm{~nm}$, respectively. The spectra of the trilayer structure with the same acceptor-Au nanosphere separation but varying donor-Au nanosphere separations of $3 \mathrm{~nm}$ (black line), $6 \mathrm{~nm}$ (dark grey line) and $9 \mathrm{~nm}$ (light grey line). All samples have $c_{D o n}=(2.8 \pm 0.2) \times 10^{17} \mathrm{~m}^{-2}$ and $c_{A c c}=(0.48 \pm 0.08) \times 10^{17} \mathrm{~m}^{-2}$, respectively. The Au NP concentration is $c_{A u}=(0.13 \pm 0.03) \times 10^{17} \mathrm{~m}^{-2}$. Schematics of the structures are also shown beside each curve.

The $\mathrm{Au}$ nanosphere concentration and the acceptor-Au nanosphere separation are two further parameters that can be tuned to optimize the acceptor emission. Fig. 3 shows the impact of these parameters not only on the acceptor emission but on the nonradiative energy transfer efficiency. The figures show the acceptor PL ratio, $I_{A c c-T r i} / I_{A c c}$, where $I_{A c c-T r i}$ is the acceptor emission in the trilayer and $I_{A c c}$ is the acceptor monolayer emission in the reference bilayer. Firstly, in Fig. 3(a) it can be seen that as the Au nanosphere concentration increases the energy transfer efficiency increases. However, the acceptor emission shows a very different dependence, increasing to a peak vale at an intermediate Au nanosphere concentration before decreasing again. This behavior is due to the competition between the increase in the acceptor emission due to the plasmon-enhanced energy transfer and increase in the direct quenching of the emission as the $\mathrm{Au}$ nanosphere concentration increases. Further details on the $\mathrm{Au}$ nanosphere concentration dependent emission quenching can be found in references 19 and 20. A similar picture is revealed in Fig. 3(b). The plasmon-enhanced nonradiative energy transfer efficiency is highest at the smallest acceptor-Au nanosphere separation and decreases as the separation increases. An efficiency of $\sim 40 \%$ is observed when both the donor and acceptor monolayers are $3 \mathrm{~nm}$ from the Au nanosphere layer. However, the acceptor emission does not show largest enhancement where the efficiency is highest but rather at larger separations of $\sim 12 \mathrm{~nm}$. Again, this behavior can be explained by the competition between the direct quenching of the acceptor emission by the Au nanospheres and the plasmon-enhanced energy transfer. ${ }^{14}$

The separation dependence also provides an opportunity to study the extension of the energy transfer distance through the interaction with the plasmons. It has been previously shown that nonradiative energy transfer in QD systems is well described by Förster resonance energy transfer and the efficiency for energy transfer from a donor QD to a plane of acceptor QDs is given by 


$$
\eta=\left[1+\frac{2 d^{4}}{c_{A c c} \pi R_{0}^{6}}\right]^{-1}
$$

where $d$ is the donor-acceptor center-to-center distance, $R_{0}$ is the Förster radius, and $c_{A c c}$ is the acceptor QD concentration. ${ }^{21,22}$ The Förster radius is the characteristic distance at which the energy transfer efficiency is $50 \%$ for a donor-acceptor pair. It can be calculated from the spectral properties of the QDs using

$$
R_{0}=0.0211\left(\frac{\kappa^{2} Q Y}{n^{4}} J\right)^{1 / 6}
$$

where $\kappa^{2}=2 / 3$ for the randomly oriented QD dipoles, $n=1.5 \pm 0.3$ is the volume weighted refractive index of the surrounding medium, $Q Y=3 \%$ is the donor QDs quantum yield and $J$ is the spectral overlap between the donor emission and acceptor absorption. The spectral overlap is calculated using

$$
J=\int_{0}^{\infty} \hat{I}_{D o n}(\lambda) \times \varepsilon_{A c c}(\lambda) \lambda^{4} d \lambda
$$

where $\hat{I}_{D o n}(\lambda)$ is the area normalized donor emission and $\varepsilon_{A c c}(\lambda)$ is the acceptor absorption. A Förster radius of $4.2 \mathrm{~nm}$ is calculated for the donor and acceptor QDs in this study. Using equation (2) the distance dependence of this nonradiative energy transfer efficiency is calculated and shown in Fig. 3(b). By comparison with the experimentally measured nonradiative energy transfer efficiency in the trilayer structures it can be clearly seen that plasmon-enhanced energy transfer substantially extends the energy transfer distance. To further quantify this extension the experimental data is fitted using equation (2) which yields a plasmon-enhanced Förster radius of $8.3 \mathrm{~nm}$, showing that through interaction with the localized surface plasmons an $\sim 100 \%$ increase in the characteristic energy transfer distance has been realized.
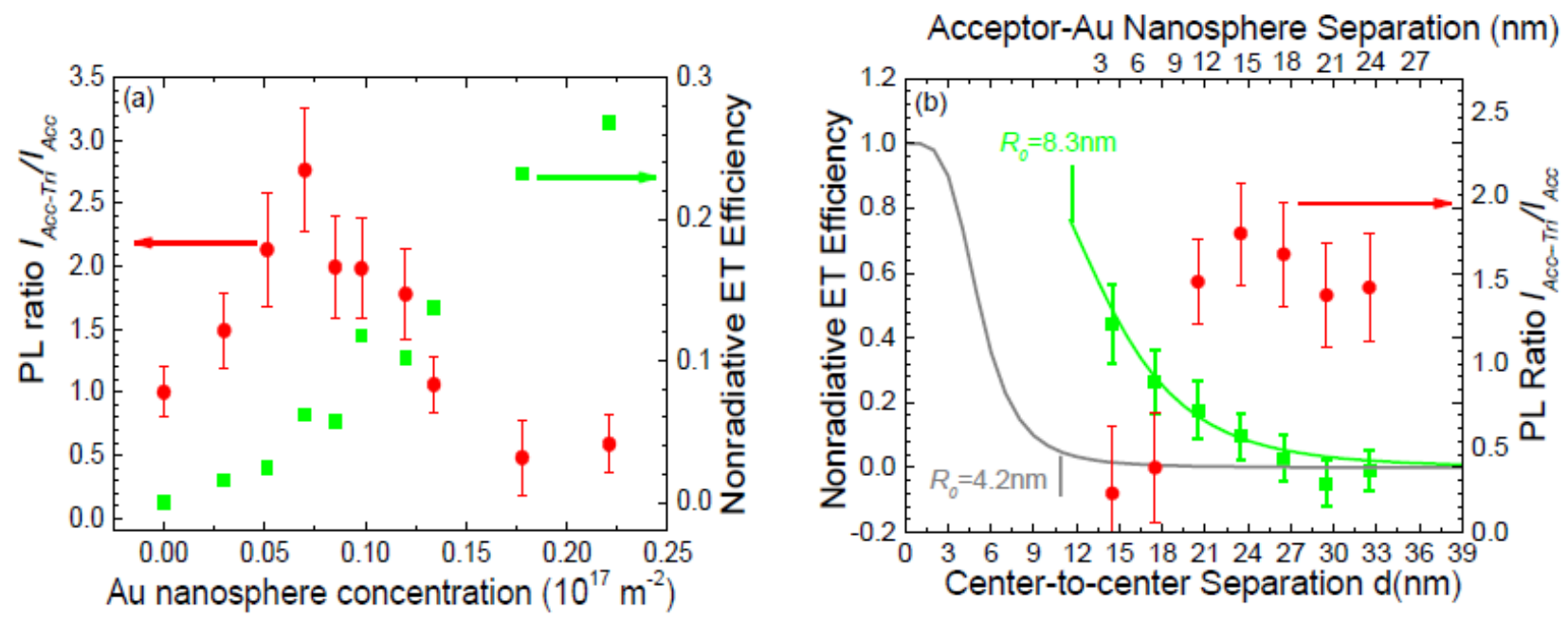

Figure 3. (a) The acceptor QD monolayer PL ratio (circles) and the plasmon-enhanced nonradiative energy transfer efficiency (squares) as a function of the Au nanosphere concentration. (b) The acceptor QD monolayer PL ratio (circles) and the plasmon-enhanced nonradiative energy transfer efficiency (squares) as a function of the acceptor-Au nanosphere separation, shown on the top axis. The bottom axis shows the corresponding center-to-center distance between the donor QD monolayer and the acceptor QD monolayer. The distance dependence of the nonradiative energy transfer in the absence of the Au nanospheres is also shown (grey line), as is the fit to the plasmon-enhanced nonradiative energy transfer dependence using equation (2) (green line).

The study of plasmon-enhanced nonradiative energy transfer in the quantum dot donor-acceptor system has highlighted clearly that the conditions for optimization of the energy transfer process and the highest acceptor emission do not necessarily correspond. Therefore, depending on the system under study and the intended application, the influence of 
the design parameters on the output through the plasmon-enhanced energy transfer and the direct interaction of the plasmonic component with the donor and acceptor emission has to be considered.

\subsection{Plasmon-mediated energy transfer from a quantum well to quantum dots}

As discussed above for many application other materials systems are required, and the donors and acceptors can have other dimensionalities Therefore it is interesting to demonstrate the plasmon-enhanced energy transfer mechanism in a hybrid structure comprised of inorganic semiconductor 1D QDs and a 2D quantum well. Previously nonradiative energy transfer from quantum wells to quantum dots and organic films has been reported. ${ }^{4,23,24}$ The $\mathrm{QW}$ structures considered to date have been limited to an acceptor QD monolayer due to the short energy transfer distances or etched structures which also aim to bring the QD acceptors closer to the QW donor. Here we report on a test structure comprised of a single InGaN QW with an emission peak at $516 \mathrm{~nm}$, overlapping the absorption of the acceptor CdSe/ZnS nanocrystal QDs emitting at $\sim 650 \mathrm{~nm}$. A GaN barrier of $3 \mathrm{~nm}$ was selected to facilitate close proximity of the donor QW and plasmonic structures. The QDs are dispersed in a $\sim 80 \mathrm{~nm}$ thick PMMA layer on top of the QW barrier. The layer has a QD concentration of $(1.9 \pm 0.1) \times 10^{21} \mathrm{~m}^{-3}$. The QW emission spectrum and QD absorption spectrum are shown in Fig. 4 (a). Finite difference time domain numerical simulations were carried out to design a plasmonic array with the extinction peak overlapping the donor emission spectrum. ${ }^{25}$ The extinction spectrum of the selected array of $100 \mathrm{~nm} \times 100 \mathrm{~nm} \mathrm{Ag}$ nanoboxes with a $100 \mathrm{~nm}$ gap is also shown. The nanoboxes have a total height of $40 \mathrm{~nm}$. This array was fabricated on top of the GaN barrier layer using He-ion lithography, and a He-ion micrograph of the array is shown in Fig. 4(b), along with a schematic of the full trilayer structure.
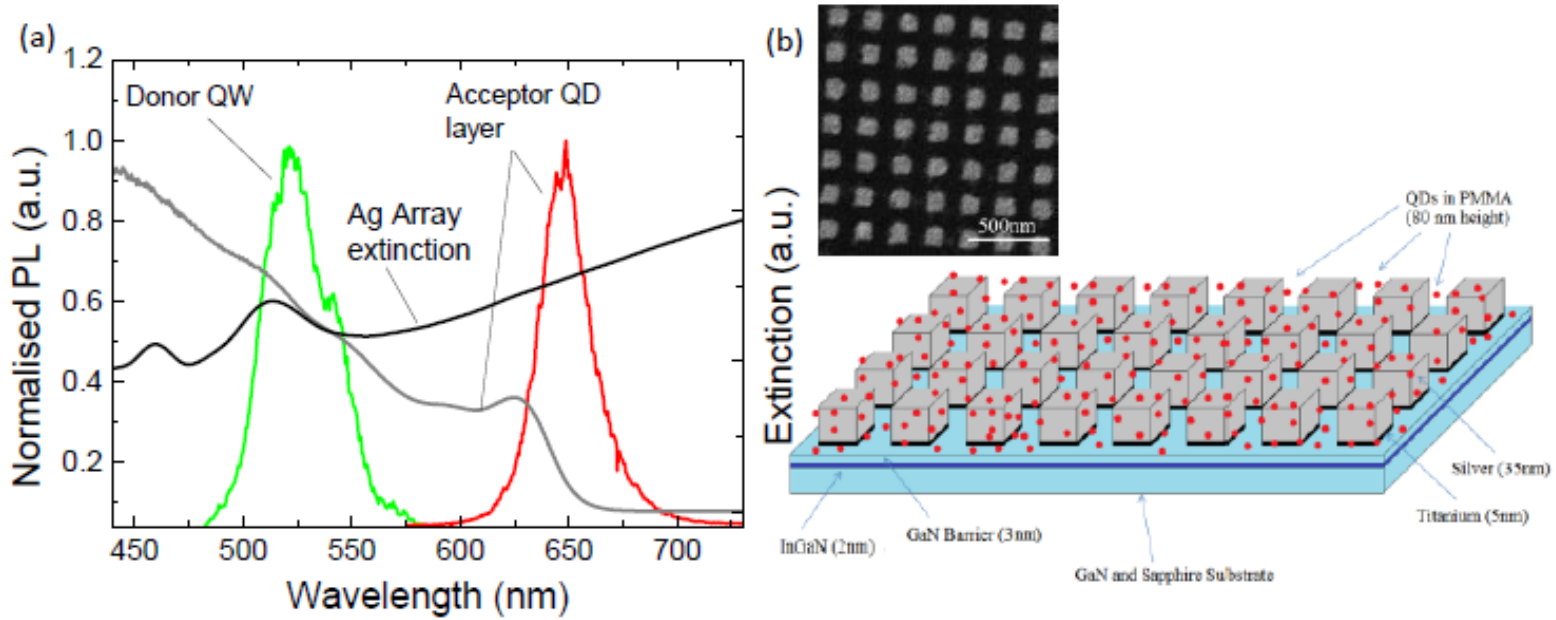

Figure 4. (a) The normalized PL spectra of the donor quantum well emission (green line) and acceptor QD layer emission (red line). The absorption spectrum of the QDs (grey line) and extinction spectrum of the Ag array (black line) are also shown. (b) Schematic of the QW-Ag array-QD trilayer showing a single InGaN QW with a $3 \mathrm{~nm}$ thick GaN barrier decorated with a Ag nanobox array, which is subsequently covered by QDs embedded in an $\sim 80 \mathrm{~nm}$ thick PMMA layer. A He-ion micrograph of the Ag nanobox array is also shown.

The QW time resolved PL decays are shown in Fig. 5(a). As for the previous all-QD structure, the direct interaction between the donor and the plasmonic structure reduces the donor lifetime and this decay becomes even faster with the addition of the acceptor QDs. In the absence of any plasmonic component there is no evidence of nonradiative energy transfer from the donor QW to the acceptor QD layer. Using equation (1) the quenching efficiency of the plasmonic array is found to be high at $\sim 90 \%$ and the plasmon-enhanced energy transfer efficiency is $\sim 27 \%$. This efficiency can be tuned by varying the plasmonic array properties and the separation between the QW and the array.

The PL spectra from the QW and QDs, with and without the Ag array is presented in Fig. 5(b). It can be clearly seen that the acceptor QD emission is higher for both samples with the array, with the highest emission for the trilayer structure. The acceptor PL enhancement is quantified using the PL ratio, $I_{A c c-T r i} / I_{A c c} \sim 1.7$, where $I_{A c c}$ is the QD emission 
directly on the QW in the absence of the plasmonic array and $I_{A c c-T r i}$ is the QD emission with the plasmonic array on top of the QW. To investigate the role of direct metal-enhanced fluorescence of the QD emission directly by the plasmonic array, the PL ratio on and off the plasmonic array fabricated on a GaN substrate is also measured. In this case it is found that the plasmomic array by itself enhances the QD layer emission by $\sim 40 \%$. The further increase observed in the trilayer structure is attributed to plasmon-enhanced nonradiative energy transfer. As discussed above, the nonradiative energy transfer efficiency does not have to be maximised to provide the largest acceptor QD emission enhancement. Furthermore, in this QW-QD structure the QDs are located around the array and not all QDs will experience the same local field. In this sense QW-QD structure is more complex to design than the all-QD structure considered earlier.
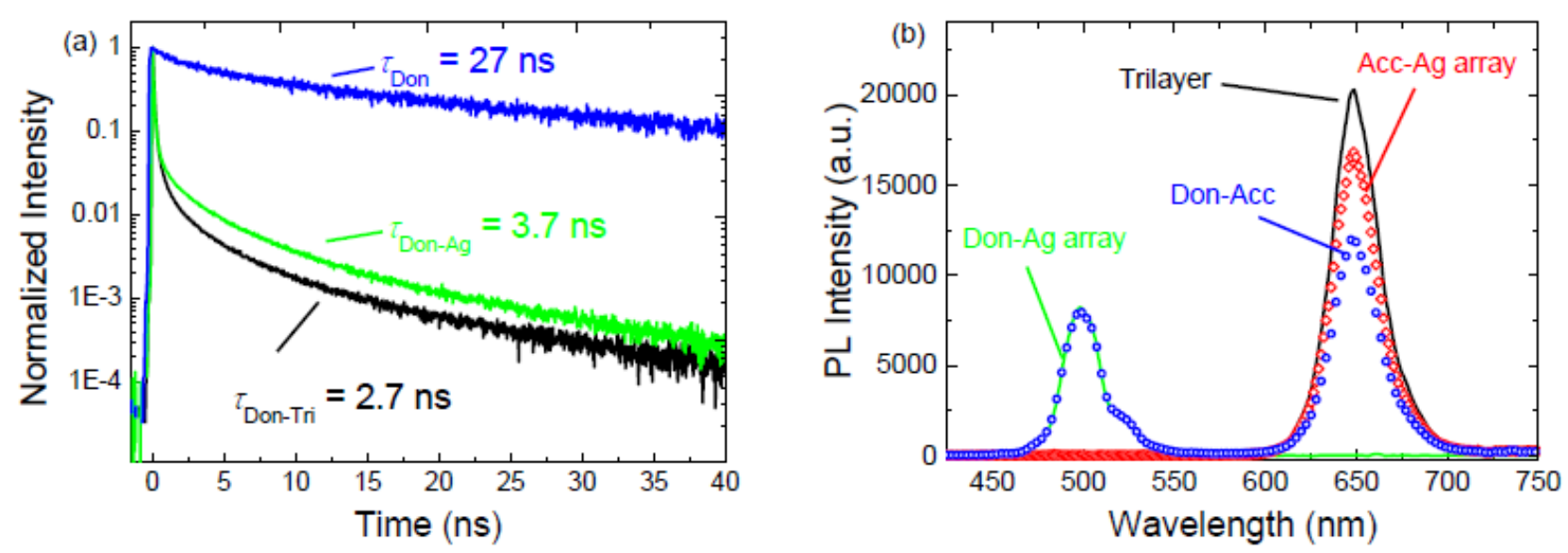

Figure 5. (a) Normalised time-resolved donor PL decays for the reference donor QW-acceptor QD structure, donor QW-Ag array structure and complete trilayer structure with lifetimes indicated by $\tau_{D o n}, \tau_{D o n-A g}$ and $\tau_{D o n-T r i}$, respectively. (b) PL spectra of reference donor QW-acceptor QD structure (blue circles), donor QW-Ag array structure (green line), acceptorQD-Ag array on GaN structure (red diamonds) and complete trilayer structure (black line).

\section{CONCLUSIONS}

We have reported on plasmon-enhanced nonradiative energy transfer in two inorganic semiconductor systems, an all-QD structure and a hybrid QW-QD structure using both colloidal and lithographically fabricated plasmonic nanostructures. Key results have been reviewed, showing that it is possible to significantly increase the energy transfer efficiency, rate and distance. It has also been shown that the conditions for maximum acceptor emission do not necessarily correspond to those for the greatest enhancement of the nonradiative energy transfer process. Careful engineering of the structures in terms of the plasmonic component properties and position relative to the donor and acceptor is required for different applications. In addition, for light emitter applications further design and experimental work can consider plasmonic arrays which can enhance the nonradiative energy transfer from the donor to the acceptor as well as promote metalenhanced fluorescence of the acceptor emission.

\section{ACKNOWLEDGEMENTS}

This work was supported by Science Foundation Ireland (SFI) under grant number 10/IN.1/12975 and the National Access Programme Grant under grant number NAP 338, and enabled using facilities funded by Irish Higher Education Authority Programme for Research in Third Level Institutions Cycles 4 and 5 via the INSPIRE and TYFFANI projects. We also acknowledge the Advanced Microscopy Laboratory (AML) for use of the helium-ion microscope. GPM acknowledges a postgraduate research scholarship from the Irish Research Council (RS/2011/287) and PJP acknowledges a SFI Engineering Professorship (SFI/07/ EN/E001A). 


\section{REFERENCES}

[1] Förster, T. “Zwischenmolekulare Energiewanderung und Fluoreszenz,” Ann. Phys. 2, 55-75 (1948).Davis, A. R., Bush, C., Harvey, J. C. and Foley, M. F., "Fresnel lenses in rear projection displays," SID Int. Symp. Digest Tech. Papers 32(1), 934-937 (2001).

[2] Stryer, L. and Haugland, R. P. "Energy transfer - a spectroscopic ruler," Proc. Natl. Acad. Sci. 58, 719-726 (1967).

[3] Yun, C. S., Javier, A., Jennings, T., Fisher, M., Hira, S., Peterson, S., Hopkins, B., Reich, N. O. and Strouse, G. F., "Nanometal surface energy transfer in optical rulers, breaking the FRET barrier," J. Am. Chem. Soc. 127, 3115-3119 (2005). Van Derlofske, J. F., "Computer modeling of LED light pipe systems for uniform display illumination," Proc. SPIE 4445, 119-129 (2001).

[4] Ackermann, M., Petruska, M. A., Kos, S., Smith, D. L., Koleske, D. D. and Klimov, V. I., "Energy-transfer pumping of semiconductor nanocrystals using an epitaxial quantum well," Nature 429, 642-646 (2004).

[5] Andrews, P. and Barnes, W. L., "Förster energy transfer in an optical microcavity," Science 290(5492), 785788 (2000).

[6] Andrews, P. and Barnes, W. L., "Energy transfer across a metal film mediated by surface plasmon polaritons," Science 306(5698), 1002-1005 (2004).

[7] Gersten, J. I. and Nitzan, A., "Accelerated energy transfer between molecules near a solid particle," Chem. Phys. Lett. 104, 31-37 (1984).

[8] Govorov, A. O., Lee, J. and Kotov, N. A., "Theory of plasmon- enhanced Förster energy transfer in optically excited semiconductor and metal nanoparticles," Phys. Rev. B 76, 125308 (2007).

[9] Rose, A., Hoang, T. B., McGuire, F., Mock, J. J., Ciracì, C., Smith,D. R. and Mikkelsen, M. H., "Control of radiative processes using tunable plasmonic nanopatch antennas," Nano Lett. 14, 4797-4802 (2014).

[10] Lessard-Viger, M., Rioux, M., Rainville, L. and Boudreau, D., "FRET enhancement in multilayer core-shell nanoparticles," Nano Lett. 9, 30663071 (2009).

[11] Ghenuche, P., de Torres, J., Moparthi, S. B., Grigoriev, V. and Wenger, J., "Nanophotonic enhancement of the Förster resonance energy-transfer rate with single nanoapertures," Nano Lett. 14(8), 4707-4714 (2014).

[12] Komarala, V. K., Bradley, A. L., Rakovich, Y. P., Byrne, S. J., Gun'ko, Y. K. and Rogach, A. L., "Surface plasmon enhanced Förster resonance energy transfer between the CdTe quantum dots," Appl. Phys. Lett. 93, 123102 (2008).

[13]Zhang, J., Fu, Y. and Lakowicz, J. R. "Enhanced Förster resonance energy transfer (FRET) on a single metal particle," J. Phys. Chem. C 111, 50-56 (2007).

[14]Zhang, X., Marocico, C. A., Lunz, M., Gerard, V. A., Gun'ko, Y. K., Lesnyak, V., Gaponik, N., Susha, A. S., Rogach, A. L. and Bradley, A. L., "Experimental and theoretical investigation of the distance dependence of localized surface plasmon coupled Förster resonance energy transfer," ACS Nano 8(2), 1273-1283 (2014).

[15] Ozel, T., Martinez, P. L. H., Mutlugun, E., Akin, O., Nizamoglu, S., Ozel, I. O., Zhang, Q., Xiong, Q. and Demir, H. V., "Observation of selective plasmon-exciton coupling in nonradiative energy transfer: donorselective $v s$ acceptor-selective plexcitons,” Nano Lett. 13, 3065-3072 (2013).

[16] Marocico, C.A., Zhang X. and Bradley, A.L., "A theoretical investigation of the influence of gold nanosphere size on the decay and energy transfer rates and efficiencies of quantum emitters," J. Chem. Phys. 144, 024108 (2016).

[17] Rogach, A. L., Franzl, T., Klar, T. A., Feldmann, J., Gaponik, N., Lesnyak, V., Shavel, A., Eychmüller, A., Rakovich, Y. P. and Donegan, J. F., "Aqueous synthesis of thiol-capped CdTe nanocrystals: State-of-the-Art," J. Phys. Chem. C 111, 14628-14637 (2007).

[18]Lunz, M., Bradley, A. L., Gerard, V. A., Byrne, S. J., Gun'ko, Y. P., Lesnyak, V. and Gaponik, N., "Concentration dependence of Förster resonant energy transfer between donor and acceptor nanocrystal quantum dot layers: effect of donor-donor interactions," Phys. Rev. B 83, 115423 (2011).

[19]Zhang, X. Marocico, C. A., Lunz, M., Gerard, V. A., Gun'ko, Y. K., Lesnyak, V., Gaponik, N. Susha, A. S., Rogach, A. L. and Bradley, A. L., "Wavelength, concentration, and distance dependence of nonradiative energy transfer to a plane of gold nanoparticles," ACS Nano 6(10), 9283-9290 (2012).

[20]Lunz, M., Zhang, X., Gerard, V. A., Byrne, S. J., Gun'ko, Y. P., Lesnyak, V., Gaponik, N., Susha, A. S., Rogach, A. L. and Bradley, A. L., "Effect of metal nanoparticle concentration on localised surface plasmon mediated Förster resonant energy transfer,” J. Phys. Chem. C 116, 26529-26534 (2012). 
[21]Lunz, M., Bradley, A. L., Chen, W.-Y. and Gun'ko, Y. K., "Förster resonant energy transfer in quantum dot layers," Superlattices Microstruct. 47, 98 (2010).

[22] Kim, D. G., Okahara, S., Nakayama, M. and Shim, Y. G., "Experimental verification of Förster energy transfer between semiconductor quantum dots," Phys. Rev. B 78, 153301 (2008).

[23] Chanyawadee, S., Lagoudakis, P. G., Harley, R. T., Charlton, M. D. B., Talapin, D. V., Huang, H. W. and Lin, C. H., "Increased color-conversion efficiency in hybrid light-emitting diodes utilizing non-radiative energy transfer," Adv. Mater. 22, 602-606 (2010).

[24] Itskos, G., Heliotis, G., Lagoudakis, P. G., Lupton, J., Barradas, N. P., Alves, E., Pereira, S., Watson, I. M., Dawson, M. D., Feldmann, J., Murray, R. and Bradley, D. D. C., "Efficient dipole-dipole coupling of MottWannier and Frenkel excitons in (Ga,In)N quantum well/polyfluorene semiconductor hetero structures," Phys. Rev. B 76, 035344 (2007).

[25] Higgins, L. J., Karanikolas, V. D., Marocico, C. A., Bell, A. P., Sadler, T.C., Parbrook, P. J. and Bradley, A.L., "Carrier density dependence of plasmon-enhanced nonradiative energy transfer in a hybrid quantum wellquantum dot structure,” Optics Express 23(2), 1377-1387 (2015). 